\title{
Balancing data on proteochemometrics activity classification
}

\author{
Angela Lopez-del Rio, ${ }^{*} \dagger$ Sergio Picart-Armada ${ }^{\dagger}{ }^{\dagger}$ and Alexandre Perera-Lluna ${ }^{\dagger, \ddagger}$ \\ $\dagger$ †2SLab, Departament d'Enginyeria de Sistemes, Automàtica i Informàtica Industrial, \\ Universitat Politècnica de Catalunya, 08028 Barcelona, Spain \\ $\ddagger$ Department of Biomedical Engineering, Institut de Recerca Pediàtrica Hospital Sant Joan \\ de Déu, Esplugues de Llobregat, 08950, Spain \\ E-mail: angela.lopez.del.rio@upc.edu
}

\begin{abstract}
In silico analysis of biological activity data has become an essential technique in pharmaceutical development. Specifically, the so-called proteochemometric models aim to share information between targets in machine learning ligand-target activity prediction models. However, bioactivity datasets used in proteochemometrics modeling are usually imbalanced, which could potentially affect the performance of the models. In this work, we explored the effect of different balancing strategies in deep learning proteochemometric target-compound activity classification models while controlling for the compound series bias through clustering. These strategies were: (1) no_resampling, (2) resampling_after_clustering, (3) resampling_before_clustering and (4) semi_resampling. These schemas were evaluated in kinases and GPCRs from BindingDB. We observed that the predicted proportion of positives was driven by the actual data balance in the test set. Additionally, it was confirmed that data balance had an impact on the performance estimates of the proteochemometrics model. We recommend a combination of data augmentation and clustering in the training set (semi_resampling) in order to
\end{abstract}


mitigate the data imbalance effect in a realistic scenario. The code of this analysis is publicly available at https://github.com/b2slab/imbalance_pcm_benchmark.

\section{Introduction}

The discovery, design and bring-to-market of a novel small-molecule drug is a very challenging process, and very expensive in terms of money, time and effort. ${ }^{1}$ Computer-Assisted Drug Design (CADD) methods can help to improve and refine the identification of hits in the first steps of drug development, thus having a huge positive impact on the costs of the whole process. ${ }^{2}$ Traditionally, interactions between ligands and targets have been predicted in CADD through a Quantitative Structure-Activity Relationship (QSAR) approach. ${ }^{3}$ In QSAR, a target is fixed and only information from compounds is used for modeling and predicting binding for said target. However, the compartmentalized nature of QSAR does not allow for discovering new cross-interactions between ligand and targets for which no training data is available. ${ }^{2}$ Proteochemometrics modeling (PCM) is an extension of QSAR which overcomes this drawback by combining information of both ligand and protein descriptors on a supervised prediction model. PCM allows for the integration of different sources of data in one model and for the general prediction of which ligands will bind to which targets. ${ }^{4}$

Both PCM and QSAR usally apply machine learning (ML) techniques such as random forests, support vector machine, logistic regression or partial least squares. ${ }^{2,4}$ Following the trends in other fields and the growing availability of data, deep learning (DL) has also been increasingly and succesfully applied on bioactivity prediction, ${ }^{5}$ specially on QSAR

modeling. ${ }^{6}$ The application of DL to PCM followed, taking advantage of public databases ${ }^{7-9}$ and improving the descriptor representation. ${ }^{10,11}$

However, an important issue for PCM and QSAR DL models is the amount and quality of data when compared to other fields of application, since increasing the number of data samples in drug discovery is expensive and thus, often infeasible. ${ }^{12}$ This poses a problem, 
since neural networks require a large quantity of training data in order to actually learn. While in other fields this problem is alleviated through data augmentation, i.e. an artificial increase of the number of observations of the training set to help the model generalize, this regularization technique is not yet commonly used in CADD. Some studies have considered different variants of the SMILES of each molecule as a way of data augmentation, ${ }^{13,14}$ but despite its proven benefits, its use is not widespread yet. This is partly due to the lack of consensus in the input representations, where alternatives to SMILES are often used.

Another factor highly affecting QSAR and PCM models is data imbalance, since the class definitions based on bioactivity data can result in highly skewed labels. In this regard, Zakharov et $\mathrm{l}^{15}$ explored how data balancing affected self-consistent regression QSAR models using highly imbalanced PubChem bioassays. The study proposed a method including costsensitive learning and under-sampling approaches to obtain more accurate predictions. Using the same data, Korkmaz explored how data balancing affected DL-based QSAR models. ${ }^{16}$ The study concluded that imbalance has indeed a negative impact on the performance of the models, but that this impact could be alleviated by applying oversampling methods like SMOTE (Synthetic Minority Oversampling Technique) ${ }^{17}$ on the fingeprint representations of the molecules. Besides, oversampling methods could also serve the purpose of augmenting the original dataset.

While the effect of data imbalance on model performance has been studied for shallow ML and DL QSAR, up to our knowledge, there are not analogous studies yet for PCM. In PCM, modeling information between targets is shared, which may compensate those for which activity data is very imbalanced. However, it is still to be proved if this compensation does happen or if the results are actually dominated by the original imbalance of each target.

Recently it has been shown that for the validation of PCM models, it is important to control the chemical series bias through clustering techniques in order to get more reliable performance estimates. ${ }^{8,18}$ This adds a complexity layer to the imbalance handling, since clustering can affect the data balance in PCM. Since Korkmaz and Zakharov et al did 
not consider the potential similarity between different compounds when validating their results, ${ }^{15,16}$ its impact on data balancing is yet to be tested.

In this paper, we study the effect of different balancing strategies in DL-based PCM target-compound activity classification models. While handling data imbalance, we also study how to integrate the compounds clustering in this process. We describe the behavior of model predictions and performance according to imbalance handling.

\section{Materials and methods}

\section{Data}

We evaluated the different balancing models on the benchmark dataset used in DeepAffinity. ${ }^{19}$ The original dataset contains binding data from BindingDB, ${ }^{20}$ merged with the amino acid sequence information from UniRef ${ }^{21}$ and the SMILES representation of compounds from STITCH. ${ }^{22}$ The original dataset consisted on IC50, $K_{i}$ or $K_{d}$ values from 829,033 compoundprotein pairs. We classified the dataset proteins into the main protein families according to the release 2018_09 from Uniprot ${ }^{23}$ and restricted our study to proteins of the kinase and G protein-coupled receptors families (separately). Binding activities were in logarithm form, so a threshold of 6 was applied in order to have binary labels for classification (active/inactive). Table 1 summarizes the final dataset we used in our analysis. The same descriptive table, but for GPCR family, can be seen in Table S1 of the Supporting Information.

Table 1: Summary of the kinases subdataset.

\begin{tabular}{ll}
\hline Entity & Number \\
\hline Compounds & 84,643 \\
Targets & 490 \\
Ligand-target pairs & 129,997 \\
$\quad$ Actives & 99,158 \\
$\quad$ Inactives & 30,839 \\
\hline
\end{tabular}

In Figures S1-S4 from the Supporting Information, the proportion of actives/inactives 
for each protein of the kinase and GPCRs protein families is represented in more detail.

\section{Descriptors}

We represented compounds by their molecular fingerprints, in which structural information is represented by bits in a bit string. We used the fingerprints from PubChem ${ }^{24}$ provided in DeepAffinity. ${ }^{19}$ In these, basic substructures of compounds are encoded in a 1D binary vector with a length of 881 bits.

We represented proteins by raw amino acid sequences transformed to one-hot encoding. Each amino acid was represented by a binary vector of length 26. Protein sequences were then normalized to the maximum length of 1499. Those sequences shorter than 1499 were zero-padded. According to the recommendation of our previous work ${ }^{25}$ we tuned the padding type and obtained the best results with pre-padding (adding zeros to the beginning of the sequence).

\section{Validation strategy}

A splitting strategy based on compound clustering (both of actives and inactives) was applied to the bioactivity data, omitting target information. Clustering-based validation strategies have been used to avoid the compound series bias, making sure that there are no similar molecules both in training, validation and test sets. ${ }^{18,26,27}$ We followed the implementation of our previous study on cross-validation strategies in $\mathrm{PCM},{ }^{8}$ where $\mathrm{K}$-means clustering with $k=100$ was applied to the fingerprint description of the compounds. Data was divided in training, validation and test sets with a proportion of $80 / 10 / 10 \%$. This splitting was randomly performed 10 times (folds) in order to test the consistency of the results, thus training and testing each model in 10 different data partitions. As further explained in the next subsection, for some balancing strategies the clustering was applied before the resampling and for others it was applied afterwards. 


\section{Balancing strategies}

We chose an oversampling method to balance data since oversampling was shown to improve performance in the Korkmaz study of data imbalance in DL-based QSAR ${ }^{16}$ and in a systematic study of data imbalance with CNNs. ${ }^{28}$ Oversampling methods increase the number of samples in the minority class to create a balanced data set. Specifically, we used the SMOTE oversampling technique, ${ }^{17}$ which creates synthetic data points of the minority class similar to those available. Resampling with SMOTE was done in a per protein basis, so that each protein would be balanced. Some proteins had to be discarded in certain strategies, since there were either only active or inactive ligands, or the number of samples in the minority class was smaller than the number of neighbors used for constructing the synthetic samples $(k=5)$ and SMOTE was not applicable.

Unlike Korkmaz, that applied data balancing methods to each training set, ${ }^{16}$ we tested four different combinations of balancing, data clustering and splitting (see Figure 1): no_resampling, in which bioactivity data for each protein was taken as it was, and clustering was applied in order to perform the splitting; resampling_after_clustering, in which after clustering data and splitting it into training, validation and test, each protein activity data in each set was resampled and attained a 50\% actives/inactives proportion; resampling_before_clustering, in which, opposite to the previous strategy, resampling was applied prior to clustering and splitting, so while the global protein-wise proportion of actives/inactives was 50\%, it did not have to be 50\% within each splitting set; and semi_resampling, in which the splitting performed in the no_resampling strategy was reused, the test set was kept without resampling but the training + validation set was resampled, re-clustered and re-splitted into train and validation.

\section{Prediction Models}

We built a DL model for studying the impact of different data balancing strategies in state-ofthe-art PCM. A random prediction was generated to have an absolute, input-naïve baseline 
to compare our results with.

\section{Random baseline}

A random baseline was computed according to the actives/inactives ratio of the training set for each strategy and each fold. Let $f$ be the fraction of actives in the training samples involving a protein, and $n$ the number of samples to be predicted in the test set for that protein. The random baseline is obtained by first sampling $\lfloor f n+0.5\rfloor$ values from a uniform distribution in $[0.5,1]$ (actives) and $n-\lfloor f n+0.5\rfloor$ values from a uniform distribution in $[0,0.5]$ (inactives), then concatenating both and shuffling. This procedure keeps the active/inactive balance by design while producing random activity predictions.

\section{Deep Learning Model}

We studied the impact of data balancing strategies on a DL model. We followed the Korkmaz strategy of selecting a simple, well-established architecture whose complexity issues would not be a confounder of the factor under study. ${ }^{16}$ We refrained from using Long ShortTerm Memory networks since they have convergence issues when training sequences longer than 1000 elements. ${ }^{29}$ Model hyperparameters were tuned using the validation set, choosing the simplest working architecture. As in our previous work, ${ }^{8}$ the DL PCM model consisted of two analysis blocks. The amino acid sequence analysis block was a 1D convolutional neural network. The fingerprints analysis block consisted of a feed-forward neural network. Dropout was used in both branches to prevent overfitting. ${ }^{30}$ The representations built by the compound and target analysis blocks were then merged and the information was passed through a softmax activation unit, which quantified the ligand-target pair activity probability. A schematic representation of the DL-based PCM model can be found in Figure S5 of the Supporting Information, along with further details on the optimised hyperparameters. 


\section{Implementation}

We trained every model with an Adam optimizer ${ }^{31}$ (learning rate $=5 \times 10^{-4}, \beta_{1}=0.1$, $\beta_{2}=0.001, \epsilon=1 \times 10^{-8}$ and decay rate defined as the learning rate/number of epochs) for 100 epochs, with a batch size of 128 both for training and validation. Models were implemented in Python 3.6.9 (Keras $^{32}$ 2.3.1 using Tensorflow ${ }^{33} 2.1 .0$ as backend) and run on two NVIDIA GeForce GTX 1070 GPUs. SMOTE data balancing was applied using the imbalanced-learn Python package. ${ }^{34}$ The statistical processing of results was performed in R software (3.6.3). ${ }^{35}$

\section{Characterization of data balance}

The data balancing strategy had an impact on the actual data balance, defined as the proportion of active molecules for a protein.

Data balance $($ protein $)=$ Proportion of actives $($ protein $)=\frac{n \_ \text {active_compounds }}{n \_t o t a l \_c o m p o u n d s}$

Thus, a comprehensive analysis of data balance was carried to better understand and interpret performance results. For each of the balancing strategies, the original distribution of active ratios per protein was characterized. We also compared the original imbalance of the training and test sets for each strategy to explore possible trends, and studied the effect that other covariates (the protein length and the number of interactions of each protein in its corresponding set and fold) might have on the original test set imbalance.

The next key question was to narrow down the factor driving the proportion of actives in the predicted data (as opposed to the original data). The main options under consideration were: (1) a constant, global imbalance that the model would learn from the whole dataset; (2) the protein-wise imbalance that the model would learn in the training set and (3) a test set-driven imbalance, based on its actual imbalance.

In the training process, the weights of the selected model were those from the epoch with the maximum accuracy (proportion of correct predictions) on the validation set. This process was run for each strategy and fold. Then, each selected model was used to predict 
on their corresponding test set. After the binarization of the test set predictions (probability threshold of 0.5 ), the proportion of predicted actives was computed by protein and also compared to the ratios of the original test and training sets.

\section{Performance Metrics}

The resampling strategies were assessed with various performance metrics for binary classifiers and prioritisers. The selection was based on those used by Korkmaz: ${ }^{16}$ balanced accuracy, F1 score, Matthews correlation coefficient (MCC) and area under the ROC curve (AUROC). All of them are insensitive to class imbalance. In the case of F1-score, we used the macro-average, which is computed by averaging the F1-score for the active and inactive labels. Further details on the definition of these metrics can be found in the Supporting Information.

The performance metrics were computed on the predictions of each selected model in its corresponding test set. AUROC was computed from raw predicted probabilities, while F1score, balanced accuracy and MCC were derived from the binarized predictions. We tested the significance of the differences between strategies by means of nonparametric two-sided Wilcoxon test for paired samples. ${ }^{36}$

\section{Explanatory Models}

Performance metrics and predicted ratios were further described through linear models built upon the different combination of variables considered in this analysis. Our prior work in similar scopes had found them insightful, since they allow for a statistical analysis of the contribution of each factor under study. ${ }^{8,25,37}$ Each of the data points used for fitting a explanatory linear model corresponded to a different protein. Simpler claims were investigated with Pearson's r for linear correlation, using confidence intervals (CI) and p-values for significance.

On the one hand, the predicted ratio of actives $\left(r_{\text {pred }}\right)$ was modelled through the quasib- 
inomial logistic model ${ }^{38}$ in equation 2 , stratified by strategy, in order to quantify the effect of different variables of interest.

$$
r_{\text {pred }} \sim r_{\text {training }}+r_{\text {test }}+\log 10\left(n_{\text {int }}\right)+\log 10\left(n_{\text {seq }}\right)+k_{\text {fold }}
$$

Specifically, the main variables of interest in this model were the actual ratios in the training $\left(r_{\text {training }}\right)$ and in the test $\left(r_{\text {test }}\right)$ sets, both numeric between 0 and 1 . As additional covariates, the number of interactions $\left(n_{\text {int }}\right)$ and the sequence length $\left(n_{\text {seq }}\right)$ (both numerical) and the fold number $\left(k_{\text {fold }}\right.$, categorical $)$ were also included. This model was not computed for the resampling_after_clustering strategy, since the data balance (and thus, the predicted active ratio) is enforced.

On the other hand, each performance metric was explained through the linear model described by the Equation 2.

$$
\text { metric } \sim \text { strategy }+\log 10\left(n_{\text {int }}\right)+\log 10\left(n_{\text {seq }}\right)+k_{\text {fold }}
$$

The response was the quantitative metric of interest in each case (one model per metric), while strategy was categorical (no_resampling, resampling_after_clustering, resampling_before_clustering, semi_resampling). The same covariates as in Equation 1 were added.

However, before evaluating the DL model, the performance metrics of the baseline were characterised: the strategy variable was tested with a type 3 analysis of variance (ANOVA) ${ }^{39}$ in order to pinpoint the imbalance-sensitive and insensitive metrics. Metrics were called imbalance-sensitive if the imbalance-aware random baseline exhibited different performances between resampling strategies.

The imbalance insensitive metric models were fitted analogously to the baseline performance models (with Equation 2). However, to address the pitfalls of the direct comparison of metrics whose baselines might differ, imbalance sensitive performance metrics were defined 
and modelled as follows:

$$
\text { adj_metric }=\text { metric }- \text { baseline }
$$

And thus, adjusted performance metrics were also described with the Equation 2 but changing the response to adj_metric of Equation 3:

$$
\text { adj_metric } \sim \text { strategy }+\log 10\left(n_{\text {int }}\right)+\log 10\left(n_{\text {seq }}\right)+k_{\text {fold }}
$$

Note that while all the metrics but MCC were non-negative, the adjusted metrics could show negative values when the performance of the DL model was lower than that of the baseline.

Reference categories for categorical variables were no_resampling for strategy and 0 for fold. Each term of the fitted model represents the difference between its specified category and the reference category of that variable.

\section{Results}

\section{Characterization of the original data balance}

\section{Distribution of the actives ratio}

Figure 3 displays the original distribution of the actives ratio in the training and test sets. Test sets tended to magnify data imbalance, creating around $24 \%$ of the times extreme cases, i.e. all actives or all inactives, not present in the training set. Strategy-wise, no_resampling kept similar data distributions in training and test; resampling_before_clustering and semi_resampling led to a more balanced training set, but an imbalanced test set, and resampling_after_clustering only kept totally balanced proteins in both training and test sets. 


\section{Training and test imbalance comparison}

Figure 2 revealed both positive, negative and null trends between the training and test protein balances, and Table S3 of the Supporting Information quantifies these correlations. No_resampling showed a positive correlation between both (Pearson's r 95\% CI: $\left.[0.338,0.400], p<10^{-16}\right)$, i.e. proteins were prone to keep their (im)balance in training and test sets. Resampling_before_clustering showed an inverse relationship (Pearson's r 95\% CI: $\left.[-0.457,-0.398], p<10^{-16}\right)$, which was expected since this strategy started from globally balanced proteins and after the clustering, an imbalance in one direction in the training set entailed an inverse imbalance in the test set. Semi_resampling led to uncorrelated train and test balances (Pearson's r 95\% CI: $[-0.024,0.051], p=0.48$ ), expected since the training set was resampled, breaking any correlation with the test set balance. Resampling_after_clustering always kept balanced proteins, by design.

\section{Other covariates}

The effect that the number of interactions for each protein in its corresponding set and fold, and the protein length (i.e. number of amino acids) had on the test set imbalance was investigated (Figures S7-S8 and Tables S4-S5 of the Supporting Information). Proteins with greatest imbalance tended to be among those with the least interactions (Table S4: Pearson's r 95\% CI $[-0.097,-0.026], p=8.01 \cdot 10^{-4}$ for no_resampling and semi_resampling; $[-0.307,-0.240], p<10^{-16}$ for resampling_before_clustering). The sequence length had

no consistent effect on the protein imbalance (Table S5: Pearson's r 95\% CI [-0.052, 0.020], $p=0.37$ for no_resampling and semi_resampling; $[-0.082,-0.009], p=0.014$ for resampling_before_clustering).

\section{Analysis of the predicted proportions}

Figure 3 represents the ratio of predicted actives by protein and Table S6 of the Supporting Information summarizes the percentage of proteins with all actives or inactives (ex- 
treme cases). They show that no_resampling strategy was inclined to predict everything as positives ( $71.6 \%$ of the time, compared to $3.5 \%$ for predicting all negatives). Resampling_before_clustering and semi_resampling alleviated the imbalance in the predictions, but still retained a spike of proteins where all the compounds were predicted as positives (23.4\% and 29.1\%) and negatives (5.5\% and 4\%). Resampling_after_clustering kept a wide and symmetric distribution of predicted actives, with only $1.2 \%$ predicted as all actives and $0 \%$ as all inactives.

Figure 3 also puts the ratio of predicted actives in context with the original training and test ratios: the distribution was most similar to that of the test proportions to that of the training ones (except resampling_after_clustering, since those proportions were constant).

Figure 4 puts the predicted ratios in context of the training ratios and Table S7 of the Supporting Information quantifies their correlations, elucidating a variety of trends: (1) no_resampling shows a positive trend between the training and the predicted ratio (Pearson's r 95\% CI: $\left.[0.440,0.496], p<10^{-16}\right)$, but since the training and the test ratio are also positively correlated (Figure 2), the latter could be the one driving the predicted ratio of positives; (2) resampling_after_clustering had a constant training ratio, meaning that the predicted ratio was not explainable by differences in training ratios; (3) resampling_before_clustering showed instead a negative relation between the training and the predicted ratio (Pearson's r $95 \%$ CI: $\left.[-0.130,-0.058], p=3.77 \cdot 10^{-7}\right)$, but since the former and the test ratio also anticorrelated (Figure 2), the simplest explanation was that the test ratio drove the predicted test ratio; (4) semi_resampling showed no apparent correlation between the predicted ratio and the training ratio (Pearson's r 95\% CI: $[-0.029,0.045], p=0.68$ ).

The models in Equation 1 that describe the predicted ratio of actives for each balancing strategy are summarized in Tables S8-S9 of the Supporting Information. For semi_resampling and resampling_before_clustering (Table S8), the original actives ratio in the test set had a positive, significant effect on the predicted actives ratio $(\beta=0.945$ and 0.784 , both $\left.p<10^{-16}\right)$. However, the original actives ratio of the training set showed no evidence 
of affecting the predicted ratio $(\beta=0.197$ and $-0.446, p=0.73$ and 0.31$)$. Conversely, for the no_resampling strategy (Table S9), both the original training $\left(\beta=8.312, p<10^{-16}\right)$ and test ratios $\left(\beta=1.102, p=2.6 \cdot 10^{-9}\right)$ had positive, significant effects on the predicted actives ratio. In the three models, the number of interactions per protein had a significant, negative effect $\left(\beta=-0.391,-0.396\right.$ and -1.24 , all $\left.p<10^{-16}\right)$, and some of the folds entailed significant variations of the predicted ratio.

\section{Performance metrics}

\section{Baseline performance}

Figure 5 shows a fold-averaged picture of the metrics by protein and by model type (DL or input-naïve baseline). Visual inspection suggested that the F1-score, accuracy, and possibly balanced accuracy were affected by the baseline data imbalance. To quantify this finding, the model in Equation 2 was fitted to the baseline performance metrics. According to Table S10 of the Supporting Information, the strategy term was significant (type 3 ANOVA, $p<10^{-16}$, $p<10^{-16}$ and $5.61 \cdot 10^{-11}$ ) for those three metrics, and non-significant in AUROC and MCC $(p=0.91$ and 0.82$)$. Based on this, metrics were divided in two types: (1) imbalancesensitive, if the baseline was different between strategies, and (2) imbalance-insensitive, if the baseline was constant.

\section{Deep Learning model}

Figure 5 displays an overview of fold-averaged performances, where strategies are paired with their baselines. Undefined metrics in edge cases were excluded. This mainly affected AUROC, where the number of proteins with metrics dropped around 25\% for semi_resampling, resampling_before_clustering and no_resampling (Table S12 of the Supporting Information). Figure 5 brought the dilemma of direct strategy comparison with imbalance-sensitive metrics, which was especially apparent for the F1-score and its high baseline in no_resampling 
(quartiles: $Q 1=0.428$, median of $0.611, Q 3=0.756$, Table $\mathrm{S} 10$ of the Supporting Information).

Absolute, baseline-naïve performance Absolute metric models (not accounting for baselines) were fitted following Equation 2, analogously to the baseline performance models. The strategy term would always explain variance (type 3 ANOVA, p-values ranged between $2.89 \cdot 10^{-15}$ and $p<10^{-16}$, see Table S13 in the Supporting Information). The models showed different behaviour in imbalance-sensitive and insensitive metrics (Table S14 of the Supporting Information). Pairwise comparisons of the strategy term coefficients using Tukey's method would point to two apparently conflicting scenarios (Figure S12 of the Supporting Information), further confirmed when prioritizing the strategies according to their expected performance through the linear models (Figure 7 and Table S15 of the Supporting Information): (a) no_resampling was suggested the best strategy by accuracy and F1-score (95\% CI of expected performances: $[0.701,0.723]$ and $[0.754,0.779])$, but this was confounded by the fact that it also held the highest baselines, and (b) resampling_before_clustering and resampling_after_clustering kept the highest performance estimates in AUROC (95\% CI $[0.699,0.724]$ and $[0.670,0.708]), \operatorname{MCC}(95 \%$ CI $[0.244,0.268]$ and $[0.296,0.337])$ and balanced accuracy (95\% CI [0.619,0.640] and [0.634, 0.670]).

Baseline-adjusted performance A descriptive plot of the adjusted metrics (Figure 6) pointed to a different scenario than that of the the adjusted ones (Figure 5).

Again, the strategy term was always significant (type 3 ANOVA, p-values ranged between $2.78 \cdot 10^{-9}$ and $p<10^{-16}$, Table S16 of the Supporting Information). Baseline adjustment brought a unified behaviour across the models (Table S17 of the Supporting Information), further confirmed in pairwise coefficient comparison (Tukey's method, Figure S13 of the Supporting Information) and in their expected performance (Figure 7 and Table S18 of the Supporting Information): resampling_before_clustering and resampling_after_clustering had the highest performance estimates (expected improvements over baseline ranging from 
0.149 to 0.263 and from 0.143 to 0.315 in all metrics), followed by semi_resampling (0.086 to 0.146$)$ and finally by no_resampling (0.057 to 0.127$)$.

\section{GPCRs}

We repeated all the previous analysis on the GPCR family to confirm whether the claims obtained for the kinases protein family could be generalized to other families. While their active proportion distributions were not too different, GPCR proteins were more imbalanced towards the actives than kinases (Figure S3 of the Supporting Information).

The main results obtained in kinases also apply to GPCRs. The distribution of actives and the comparison between training and test set imbalances in kinases also applies to GPCRs, except for semi_resampling, where GPCRs exhibit a certain degree of positive correlation (Pearson's r 95\% CI [0.029,0.105], $p=5.91 \cdot 10^{-4}$ ) between training and test balances (see Table S19 of the Supporting Information). The effect of the number of interactions and the sequence length on the protein imbalance were replicated on the GPCRs. Kinases and GPCRs essentially agreed on the predicted actives proportions analyses, the only exception being the $\mathrm{n}$ _interactions coefficient, non-significant in the semi_resampling strategy $(\beta=-0.011, p=0.3$, Table S20 of the Supporting Information). Regarding performance, the explanatory linear models on GPCRs led to facts equivalent to those of kinases in baseline metrics, in absolute and in baseline-adjusted performance. Regarding adjusted performances, semi_resampling significantly outperformed no_resampling in 3 metrics instead of 4 (Table S21 of the Supporting Information), which still made it preferable. Supplement 3 gathers with detail all the results obtained in the analysis of the GPCR family. 


\section{Discussion}

\section{The impact of clustering in final imbalance was strategy-dependent}

This study is focused on the characterization of the data imbalance present in bioactivity datasets, as well as how to address it. Bioactivity data also poses the problem of chemical series, i.e. sets of similar molecules with similar activities, that result in inflated performance metrics when split between training and test sets. We addressed those via a clustering prior to the splitting, ensuring that similar molecules would belong to the same set.

The first observation was that clustering modified data imbalance in a strategy-dependent way. When the starting set was perfectly balanced (strategy resampling_before_clustering), clustering and splitting induced a degree of imbalance, particularly visible in the heavier tails of the active ratios distributions in the test set. Compared to training, the lower sample sizes in the test set may also cause extreme imbalances more often. On the other end, this effect was only moderate in no_resampling, where the distribution of actives ratio was similar in train and test, but that of test had more extreme proteins with either all actives or all inactives.

Besides the overall changes in data imbalance, strategies differed in how the imbalance of a certain protein in the training set would translate to the test set. The positive trend in no_resampling suggests that existing data imbalances tended to persist after the clustering and splitting. The negative trend in resampling_before_clustering hints that, in the absence of imbalance, clustering will induce it. The flat trend in semi_resampling supports that the imbalance induced with the clustering in the training set, which was balanced with SMOTE beforehand, is independent from the original imbalance in the dataset (present in the test set). 


\section{The predicted actives proportion was driven by the test set rather than the training}

The original distribution of actives ratio in each of the balancing strategies affected the predicted actives ratio by the models. Due to the lack of correlation between training and test ratios (Figure 2), the semi_resampling strategy was the ideal scenario to disentangle their effect on the predicted ratio of actives (see model in table S3 of the Supporting Information). Its additive model suggested that the original ratio of actives in test explained the predicted proportions, rather than the training ratio. We also found that the number of interactions per protein was a relevant factor: the more interactions, the less active proportion, suggesting that the extreme cases with all predicted as actives tended to be proteins with few interactions.

Likewise, resampling_before_clustering showed negative correlation between training and test ratios, also providing a reasonably good scenario to distinguish their effects (Table S3 from the Supporting Information). Its explanatory model confirmed both conclusions from the model in the semi_resampling strategy, with similar estimates (Table S8).

The explanatory model for the no_resampling strategy (Table S9 of the Supporting Information) suffered from the positive correlation between training and test ratios, which could be confounded. Both original training and test ratios showed a positive effect on the predicted fraction of actives. Although the estimate was larger and more significant for the training ratio coefficient, the confounding effect and the very skewed distribution of the predicted ratios deemed this model inconclusive.

\section{Imbalance-sensitive metrics required baseline adjustment}

The prediction task studied here posed a particular challenge: data imbalance happened on a protein basis, and the imbalance of certain proteins could be extreme (very low or high), moving away from the global actives ratio. Each resampling strategy would lead 
to different protein-wise imbalance patterns. The baseline performance of some metrics (accuracy, F1 score and balanced accuracy) was different between strategies, while it was constant for others (AUROC and MCC). The data-driven division into imbalance-sensitive and insensitive metrics was an important step to understand the opposite conclusions reached within each metric type after direct performance comparison between strategies (Figure 7).

The direct comparison of resampling strategies with imbalance-sensitive metrics would be confounded by the imbalance-induced bias in the metrics and the protein-wise imbalance differences between strategies. We found that adjusting by the baseline metrics (see Equation 4) brought an agreement in the conclusions obtained by both imbalance-sensitive and insensitive metrics. In turn, the same conclusions were obtainable by direct comparison of imbalance-insensitive metrics. Because of this, our recommendation is to include imbalance-aware baselines and to adjust imbalance-sensitive metrics when used for model selection.

\section{Augmenting the test set was the largest performance drive}

Our results showed that the largest impact in performance estimates was the application of data augmentation to the test set: resampling_before_clustering and resampling_after_clustering tended to outperform semi_resampling and no_resampling. However, augmenting the test set might not faithfully reflect new data anymore, and could artificially inflate the performance estimates: models may specialize in discriminating between original and resampled data points instead of actives and inactives.

\section{Resampling improved performance when keeping the original test set}

On the other hand, semi_resampling outperformed no_resampling in four out of five metrics (Tukey's method, $p<0.05$, Figure S13 of the Supporting Information), which supported 
data augmentation usefulness even if the data balance in the test set differed from that of the training set. This was consistent with the observation that the main influence on the predicted actives ratio in the test set were their actual ratios in the test set instead of the original ratios in the training set. Combined with the less skewed distributions of predicted active ratios of semi_resampling against no_resampling (Figure 3), we recommend semi_resampling for future studies.

\section{Using GPCRs as an external protein family dataset for validation suggests replicability of the main guidelines}

The results obtained by the kinases and the GPCR proteins, used as an external validation set for the model fitting and evaluation, point to the same general picture with aligned conclusions. The differences found (the effect of the sequence length on protein imbalance and $n$ _interactions on predicted actives proportion is different to GPCRs) could be due to the fact that there is more imbalance of the GPCRs towards the actives. However, these results lead us to think that the guidelines for proteochemometrics models of this study provide sensible defaults to more protein families.

\section{Similarities with existing literature}

In this paper we have confirmed that data balance has an impact in DL proteochemometric target-compound activity models. Zakharov et al and Korkmaz arrived to a similar con-

clusion in a QSAR setting, ${ }^{15,16}$ the latter also using DNN models for classification. More specifically, Korkmaz stated that the higher the imbalance for a protein, the worse the model performance (measured by F1-score and MCC).

These studies got the best performances by controlling data balance by means of undersampling techniques (in the case of Zakharov) and oversampling techniques (in the case of Korkmaz). We chose SMOTE for data balancing, an oversampling technique, since the settings of the Korkmaz study were more aligned with ours and because DL models require 
a large quantity of training data. Specifically, in four out of five metrics, proteins with more interactions were better predicted (table S17 of the Supporting Information) which was also found in the Korkmaz paper.

Within our resampling strategies, semi_resampling was the most similar to the balancing process in the Korkmaz study, in which the training and validation sets were oversampled (per protein) while the test set was not.

\section{Dissimilarities with existing literature}

Technical differences existed in the descriptors used in the three studies. Zakharov et al used Quantitative Neighborhood of Atoms and biological descriptors, whereas Korkmaz used the PaDEL software. We, on the other hand, used the fingeprints from PubChem. The fact that the overall messages are consistent suggests a degree of independence from the input encoding.

More importantly, Zakharov and Korkmaz studies did not take into account the control of the compound series bias. This step is necessary for obtaining realistic performance estimates in a real-world setting. ${ }^{8,18}$ Not only we accounted for it, but we also investigated if the stage in which the compound series control was introduced, in combination with the data augmentation (before or after applying SMOTE), had an impact in the outcome.

Indeed, the order had an impact in the model performance and needed careful consideration. Resampling_before_clustering solved the global imbalance of the dataset, but clustering after oversampling would lead again to a protein-wise imbalance. Analogously, semi_resampling resampled the training and validation sets, but imbalance returned after

their clustering. On the contrary, resampling_after_clustering first corrected the problem of similar compounds, and then augmented the data to reach a protein-wise balance. 


\section{Limitations and future work}

This study continues our incremental work on recommendations for DL models regarding input encoding ${ }^{25}$ and control of chemical series. ${ }^{8}$ While this study was limited to one architecture and two protein families, it provides a foundation to understand the basic behaviour of PCM models, insights on how to adjust performance metrics for a protein-wise analysis, and a first step towards exploring more general questions. Those could include architecturecentric analyses to confirm if the same trends are observed when changing the layers or the model structure, or using other protein families with a different distribution of actives ratios, which may be flat or skewed to the inactives.

\section{Conclusion}

Although the effect of data balance and resampling techniques had been analysed for QSAR models, it had not been studied yet in the context of proteochemometrics models, even if the bioactivity datasets used in this setting are usually imbalanced. In this paper, we have tested four different combinations of data oversampling (through SMOTE) and clustering for controlling compounds similarity. While the clustering avoids overly optimistic performance estimates, it could introduce more data imbalance (in the form of splittings having proteins with mostly active or inactive compounds). Despite this potential conflict between the resampling and the clustering, we found that resampling was useful to improve the model behaviour and performance.

Some common performance metrics were affected by the data imbalance and yielded misleading trends. We included an imbalance-aware random baseline and defined baselineadjusted metrics to overcome this issue, especially in F1-score and accuracy. After baseline adjustment, the metrics provided a unified picture: the largest impact in performance estimates came from the application of data augmentation to the test set (resampling_before_clustering

and resampling_after_clustering outperformed semi_resampling and no_resampling). How- 
ever, augmenting the test set may not reflect a realistic scenario.

On the other hand, semi_resampling outperformed no_resampling in four out of five adjusted metrics and provided a more equalized distribution of predicted actives ratio. This confirmed the data augmentation usefulness even if the data balance in the test set differed from that of the training set. This was consistent with the finding that the predicted proportion of positives of the proteochemometrics model was explained by the actual data balance in the test set, rather than that of the training set. We also found that proteins with more interactions were better predicted.

Our recommendation is thus to use the semi_resampling strategy, i.e. clustering compounds to separate training and validation from test sets, resampling training and validation and then clustering compounds again to definitely split training and validation sets. This was carried out on the kinases protein family and further confirmed on the GPCR family. While we cannot extrapolate these results to all the proteins and imbalance distributions, this sets a sensible starting point for improving proteochemometrics modelling and remains consistent with the corresponding data imbalance studies on QSAR models.

\section{Data and code availability}

The bioactivity data used in our analysis is publicly available in the repository https: //github.com/Shen-Lab/DeepAffinity. ${ }^{19}$ The code of this analysis is publicly available at https://github.com/b2slab/imbalance_pcm_benchmark.

\section{Acknowledgement}

This work was supported by the Spanish Ministry of Economy and Competitiveness (www.mineco.gob.es) TEC2014-60337-R, DPI2017-89827-R, Networking Biomedical Research Centre in the subject area of Bioengineering, Biomaterials and Nanomedicine (CIBER-BBN) and Share4Rare project (Grant Agreement 780262), initiatives of Instituto de Investigación Carlos III (ISCIII). 
B2SLab is certified as 2017 SGR 952.

\section{Supporting Information Available}

The following files are available free of charge.

- Supporting Information: this is the file that contains the tables and figures referenced throughout the main text. It contains additional material to support our findings, including a more detailed explanation of the material, a depiction of the deep learning model architecture, further description of the performance metrics and complementary results of our analysis in the shape of explanatory linear models tables, significance tests and descriptive figures.

- Supplement 2: model prediction and performance (kinases): Complete analysis and results for kinases.

- Supplement 3: model prediction and performance (GPCRs): Complete analysis and results for GPCRs. Affirmations coincident with those from the kinases are marked in blue. Differences/comments specific for the GPCRs are written in pink.

\section{References}

(1) DiMasi, J. A.; Grabowski, H. G.; Hansen, R. W. Innovation in the Pharmaceutical Industry: New Estimates of R\&D Costs. J. Health Econ. 2016, 47, 20-33.

(2) Qiu, T.; Qiu, J.; Feng, J.; Wu, D.; Yang, Y.; Tang, K.; Cao, Z.; Zhu, R. The Recent Progress in Proteochemometric Modelling: Focusing on Target Descriptors, Cross-Term Descriptors and Application Scope. Brief. Bioinform. 2017, 18, 125-136.

(3) Hansch, C.; Fujita, T. p- $\sigma-\pi$ Analysis. A Method for the Correlation of Biological Activity and Chemical Structure. J. Am. Chem. Soc. 1964, 86, 1616-1626. 
(4) Bongers, B. J.; Ijzerman, A. P.; Van Westen, G. J. Proteochemometrics Recent Developments in Bioactivity and Selectivity Modeling. Drug Discov. Today: Technol. 2020,

(5) Chen, H.; Engkvist, O.; Wang, Y.; Olivecrona, M.; Blaschke, T. The Rise of Deep Learning in Drug Discovery. Drug Discov. Today 2018, 23, 1241-1250.

(6) Ghasemi, F.; Mehridehnavi, A.; Pérez-Garrido, A.; Pérez-Sánchez, H. Neural Network and Deep-Learning Algorithms Used in QSAR Studies: Merits and Drawbacks. Drug Discov. Today 2018, 23, 1784-1790.

(7) Lenselink, E. B.; Ten Dijke, N.; Bongers, B.; Papadatos, G.; van Vlijmen, H. W. T.; Kowalczyk, W.; IJzerman, A. P.; van Westen, G. J. P. Beyond the Hype: Deep Neural Networks Outperform Established Methods Using a ChEMBL Bioactivity Benchmark Set. J. Cheminformatics 2017, 9, 45.

(8) Lopez-Del Rio, A.; Nonell-Canals, A.; Vidal, D.; Perera-Lluna, A. Evaluation of CrossValidation Strategies in Sequence-Based Binding Prediction Using Deep Learning. J. Chem. Inf. Model. 2019, 59, 1645-1657.

(9) Zakharov, A. V.; Zhao, T.; Nguyen, D.-T.; Peryea, T.; Sheils, T.; Yasgar, A.; Huang, R.; Southall, N.; Simeonov, A. Novel consensus architecture to improve performance of large-scale multitask deep learning QSAR models. J. Chem. Inf. Model. 2019, 59, $4613-4624$.

(10) Jiménez, J.; Skalic, M.; Martinez-Rosell, G.; De Fabritiis, G. KDEEP: Protein-ligand Absolute Binding Affinity Prediction via 1D-Convolutional Neural Networks. J. Chem. Inf. Model. 2018, 58, 287-296.

(11) Jaeger, S.; Fulle, S.; Turk, S. Mol2vec: Unsupervised Machine Learning Approach with Chemical Intuition. J. Chem. Inf. Model. 2018, 58, 27-35. 
(12) Yang, X.; Wang, Y.; Byrne, R.; Schneider, G.; Yang, S. Concepts of Artificial Intelligence for Computer-Assisted Drug Discovery. Chem. Rev. 2019, 119, 10520-10594.

(13) Kimber, T. B.; Engelke, S.; Tetko, I. V.; Bruno, E.; Godin, G. Synergy Effect Between Convolutional Neural Networks and the Multiplicity of SMILES for Improvement of Molecular Prediction. arXiv preprint arXiv:1812.04439 2018,

(14) Bjerrum, E. J. SMILES Enumeration as Data Augmentation for Neural Network Modeling of Molecules. arXiv preprint arXiv:1703.07076 2017,

(15) Zakharov, A. V.; Peach, M. L.; Sitzmann, M.; Nicklaus, M. C. QSAR Modeling of Imbalanced High-Throughput Screening Data in PubChem. J. Chem. Inf. Model. 2014, $54,705-712$.

(16) Korkmaz, S. Deep Learning-Based Imbalanced Data Classification for Drug Discovery. J Chem Inf Model 2020, 60, 4180 - 4190.

(17) Chawla, N. V.; Bowyer, K. W.; Hall, L. O.; Kegelmeyer, W. P. SMOTE: Synthetic Minority Over-Sampling Technique. J. Artif. Intell. Res. 2002, 16, 321-357.

(18) Mayr, A.; Klambauer, G.; Unterthiner, T.; Steijaert, M.; Wegner, J. K.; Ceulemans, H.; Djork-Arné, C.; Hochreiter, S. Large-Scale Comparison of Machine Learning Methods for Drug Target Prediction on ChEMBL. Chem. Sci. 2018,

(19) Karimi, M.; Wu, D.; Wang, Z.; Shen, Y. DeepAffinity: Interpretable Deep Learning of Compound-Protein Affinity through Unified Recurrent and Convolutional Neural Networks. Bioinformatics 2019, 35, 3329-3338.

(20) Gilson, M. K.; Liu, T.; Baitaluk, M.; Nicola, G.; Hwang, L.; Chong, J.; P., O.; C., S.; K., T.; B., W.; C, P.; H, B.; L, S.-T.; R, D. BindingDB in 2015: A Public Database for Medicinal Chemistry, Computational Chemistry and Systems Pharmacology. Nucleic Acids Res 2016, 44, D1045-D1053. 
(21) Suzek, B. E.; Wang, Y.; Huang, H.; McGarvey, P. B.; Wu, C. H.; the UniProt Consortium, UniRef Clusters: A Comprehensive and Scalable Alternative for Improving Sequence Similarity Searches. Bioinformatics 2014, 31, 926-932.

(22) Kuhn, M.; von Mering, C.; Campillos, M.; Jensen, L. J.; Bork, P. STITCH: Interaction Networks of Chemicals and Proteins. Nucleic Acids Res. 2007, 36, D684-D688.

(23) Consortium, T. U. UniProt: a worldwide hub of protein knowledge. Nucleic Acids Res. 2018, 47, D506-D515.

(24) Kim, S.; Chen, J.; Cheng, T.; Gindulyte, A.; He, J.; He, S.; Li, Q.; Shoemaker, B. A.; Thiessen, P. A.; Yu, B., et al. PubChem 2019 Update: Improved Access to Chemical Data. Nucleic Acids Res. 2019, 47, D1102-D1109.

(25) Lopez-del Rio, A.; Martin, M.; Perera-Lluna, A.; Saidi, R. Effect of Sequence Padding on the Performance of Protein-Based Deep Learning Models. Sci. Rep. 2020, 1-14.

(26) Rodríguez-Pérez, R.; Bajorath, J. Multitask Machine Learning for Classifying Highly and Weakly Potent Kinase Inhibitors. ACS Omega 2019, 4, 4367-4375.

(27) Ramsundar, B.; Liu, B.; Wu, Z.; Verras, A.; Tudor, M.; Sheridan, R. P.; Pande, V. Is Multitask Deep Learning Practical for Pharma? J. Chem. Inf. Model. 2017, 57, 2068-2076.

(28) Buda, M.; Maki, A.; Mazurowski, M. A. A Systematic Study of the Class Imbalance Problem in Convolutional Neural Networks. Neural Networks 2018, 106, 249-259.

(29) Li, S.; Li, W.; Cook, C.; Zhu, C.; Gao, Y. Independently Recurrent Neural Network (IndRNN): Building a Longer and Deeper RNN. Proc. CVPR IEEE. 2018; pp 54575466.

(30) Srivastava, N.; Hinton, G.; Krizhevsky, A.; Sutskever, I.; Salakhutdinov, R. Dropout: a 
Simple Way to Prevent Neural Networks from Overfitting. J. Mach. Learn. Res. 2014, 15, 1929-1958.

(31) Kingma, D. P.; Ba, J. Adam: A Method for Stochastic Optimization. arXiv preprint arXiv:1412.6980 2014,

(32) Chollet, F., et al. Keras. https://keras.io, 2015.

(33) Abadi, M. et al. TensorFlow: Large-Scale Machine Learning on Heterogeneous Systems. 2015; https://www.tensorflow.org/, Software available from tensorflow.org.

(34) Lemaitre, G.; Nogueira, F.; Aridas, C. K. Imbalanced-learn: A Python Toolbox to Tackle the Curse of Imbalanced Datasets in Machine Learning. J. Mach. Learn. Res. 2017, 18, 559-563.

(35) R Core Team, R: A Language and Environment for Statistical Computing. R Foundation for Statistical Computing: Vienna, Austria, 2020.

(36) Wilcoxon, F. Individual Comparisons by Ranking Methods; 1945; Vol. 1; pp 80-83.

(37) Picart-Armada, S.; Barrett, S. J.; Willé, D. R.; Perera-Lluna, A.; Gutteridge, A.; Dessailly, B. H. Benchmarking network propagation methods for disease gene identification. PLoS Comput. Biol. 2019, 15, 1-24.

(38) Hardin, J. W.; Hardin, J. W.; Hilbe, J. M.; Hilbe, J. Generalized Linear Models and Extensions; Stata Press, 2007.

(39) Fisher, R. A. Breakthroughs in statistics; Springer, 1992; pp 66-70. 


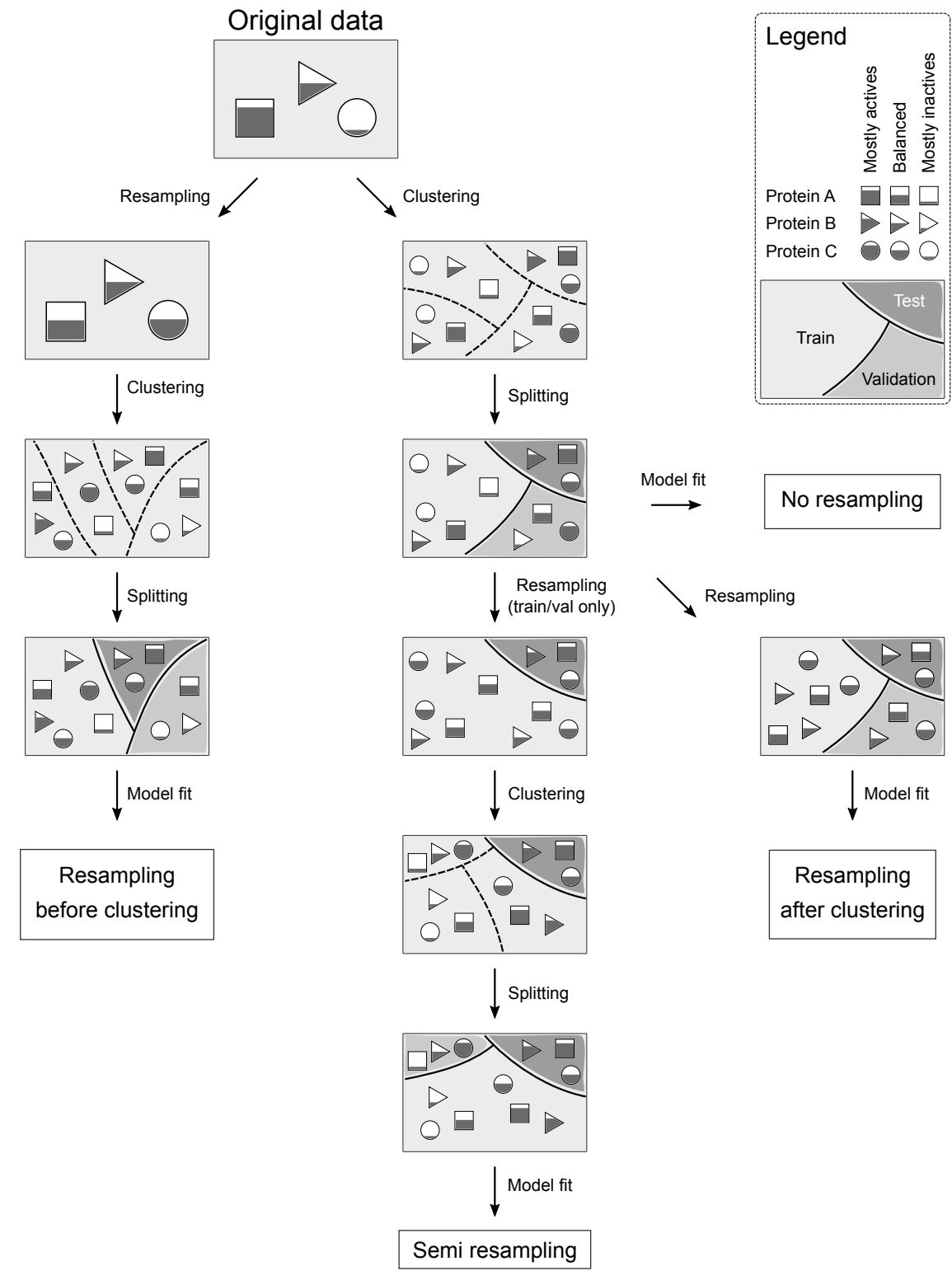

Figure 1: Description of the four balancing strategies that were applied to the bioactivity data. Resampling_before_clustering, where resampling per protein is applied prior to clustering and splitting; resampling_after_clustering, where data is first clustered and splitted and then each protein activity data in each set is resampled; semi_resampling, in which the splitting is performed and then the test set is kept without resampling but the training + validation set is resampled and clustered; and no_resampling, in which the imbalance of the original data is kept and clustering is applied prior to splitting. 


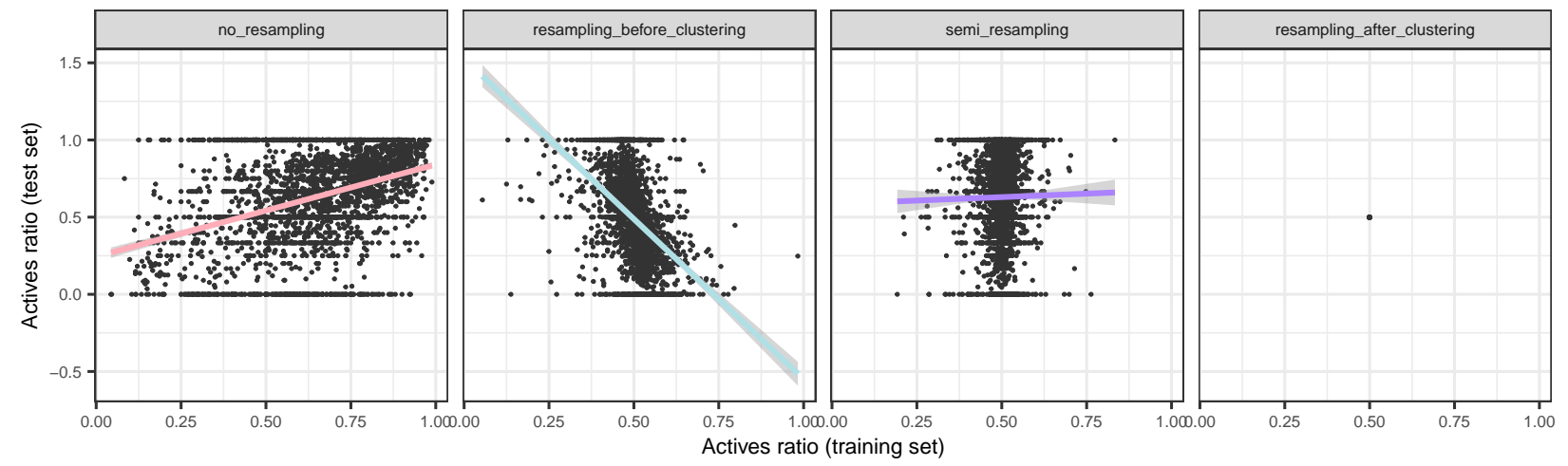

Figure 2: Comparison of the training and test original active ratios, by resampling strategy. Linear fit trends were added by strategy, and the shadowed areas indicated the $95 \%$ CI of the expected value. Each plot combines all the folds. 

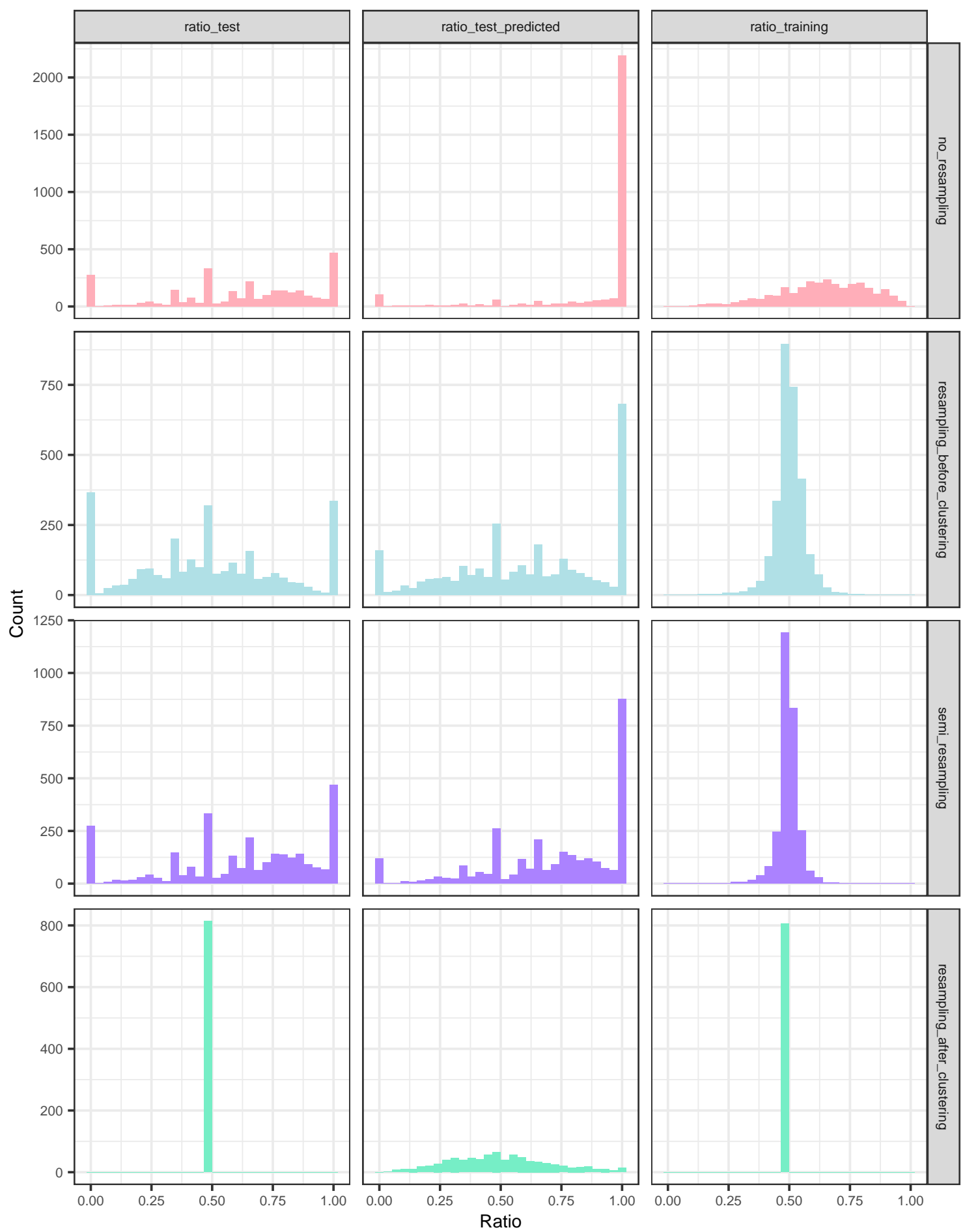

Figure 3: Histograms of the active ratios in the training set, and in the test set (both original and predicted by the deep learning model), within each resampling strategy. Each histogram combines all the folds. 


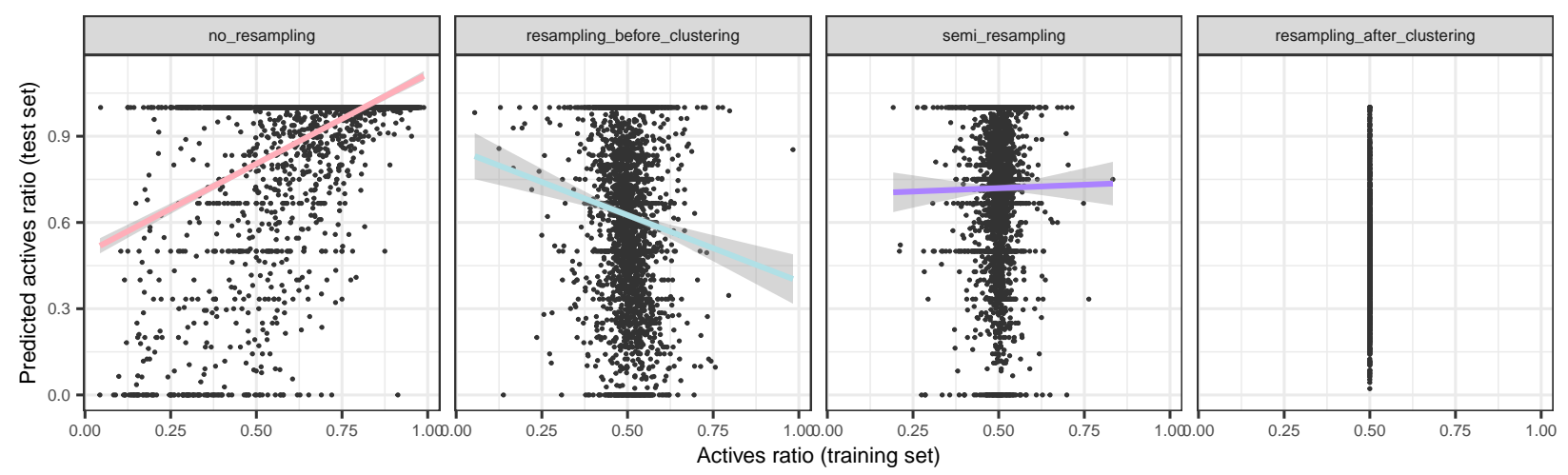

Figure 4: Predicted ratios, as a function of training ratios, by resampling strategy. Linear fit trends were added by strategy, and the shadowed areas indicated the $95 \%$ CI of the expected value. Each plot combines all the folds.
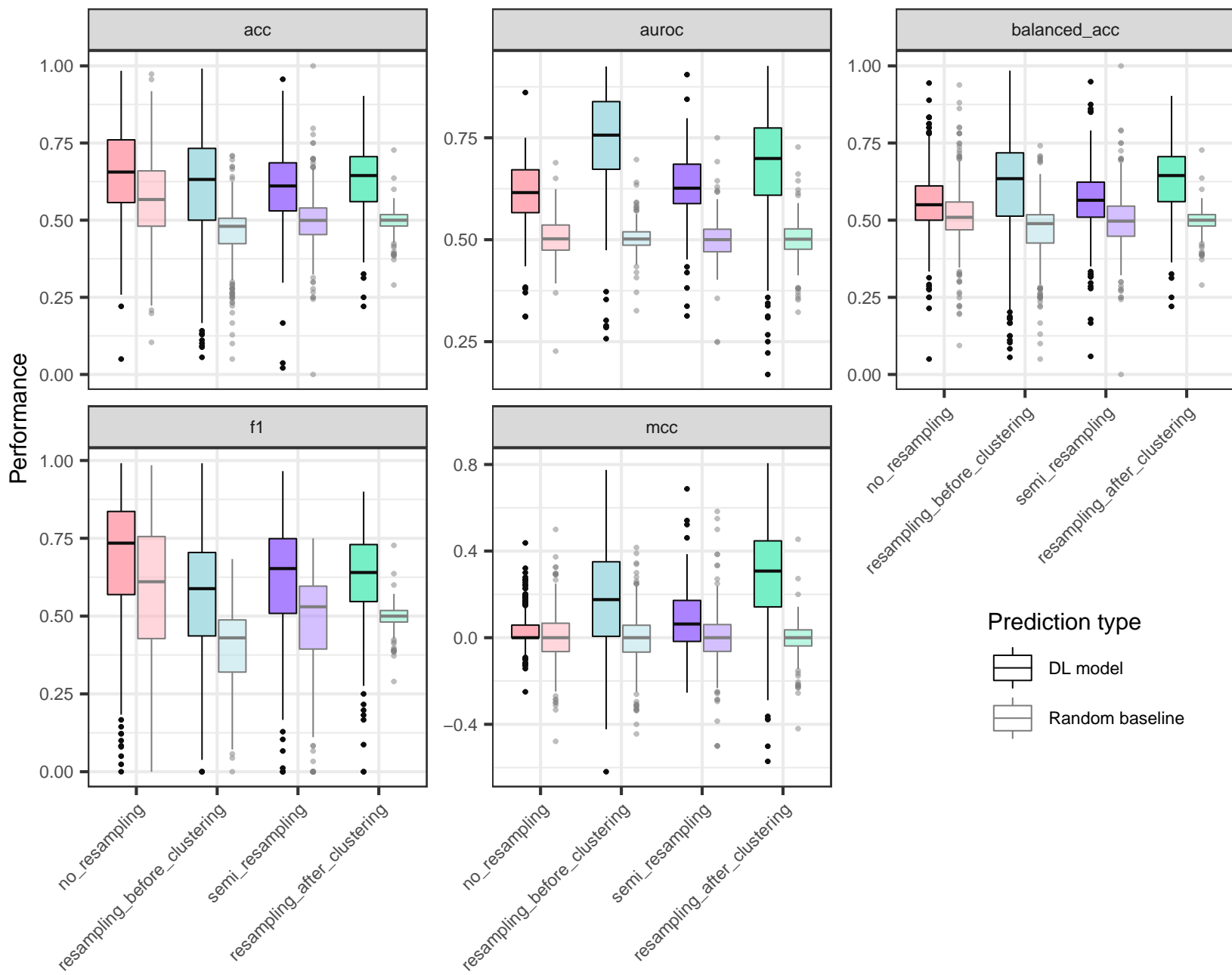

Prediction type

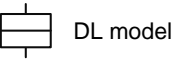

$\square$ Random baseline

Strategy

Figure 5: Absolute performance metrics for balancing strategies and their corresponding imbalance-aware random baselines. Data points correspond to proteins, averaged over folds. 

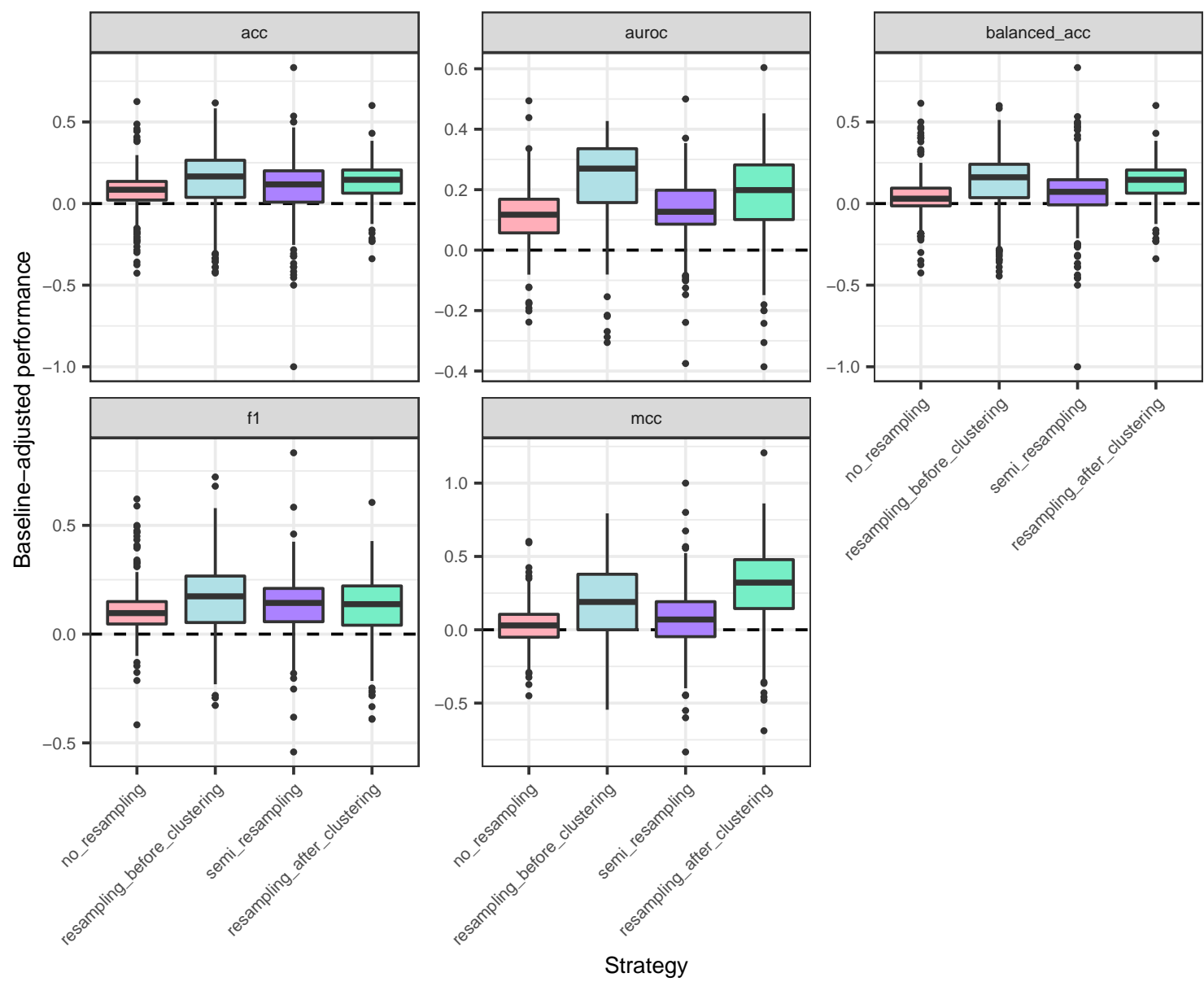

Figure 6: Baseline-adjusted performance metrics for balancing strategies. Data points correspond to proteins, averaged over folds. Values are positive when the DL model performs better than its paired imbalance-aware baseline, and negative otherwise. 


\section{Method ranking by adjusted performance.}

Lower ranks are better. Expected fold-averaged performances in brackets.
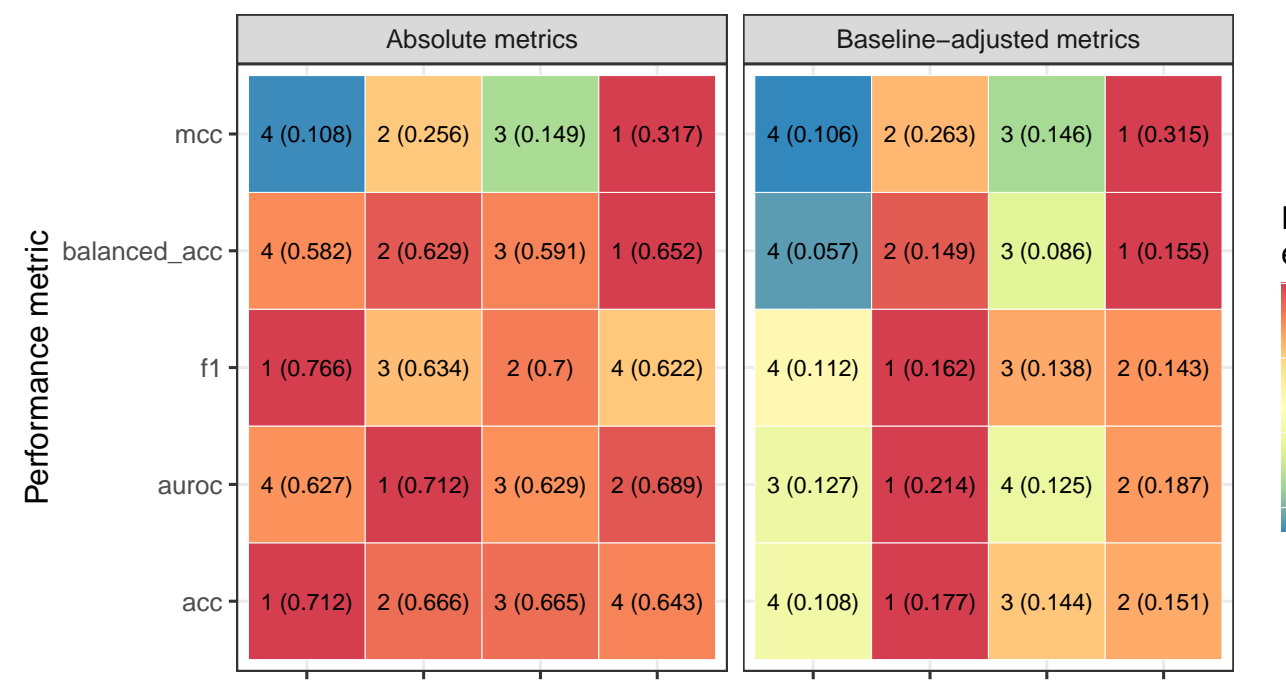

Performance rescaled by each block row maximum 1.0
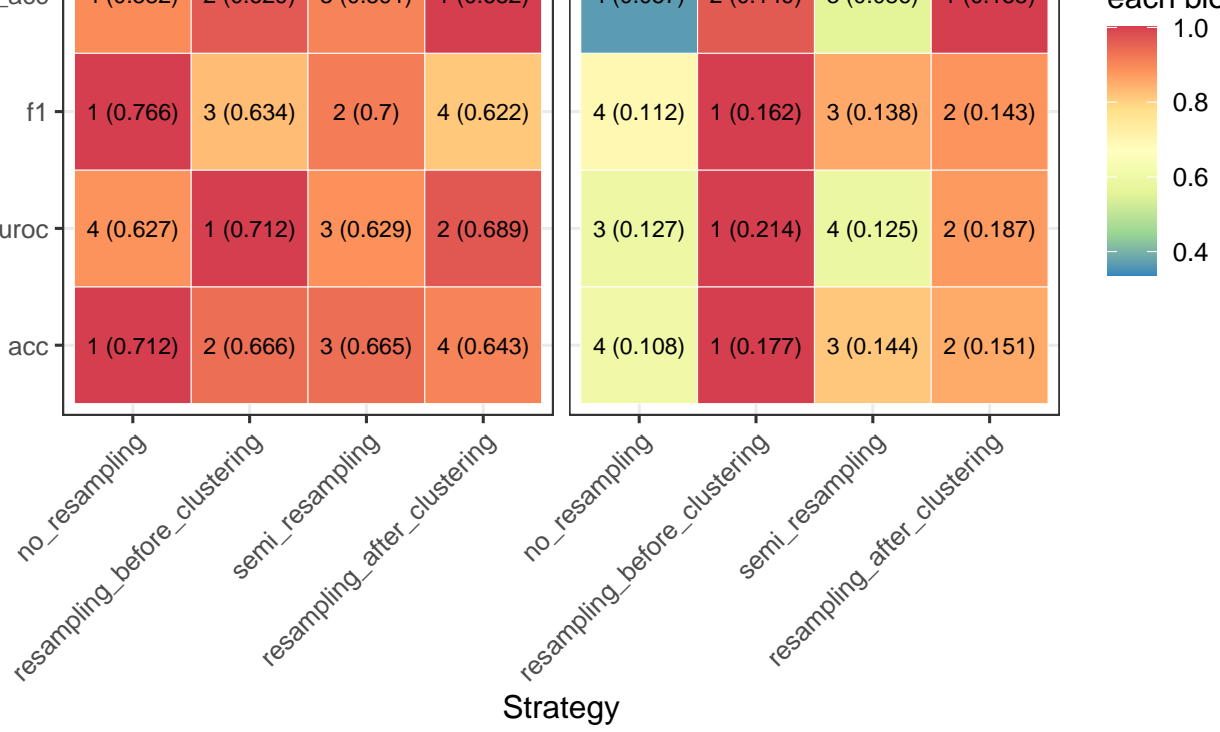

0.8

0.6

0.4

Figure 7: Resampling strategy ranking according to their absolute (left block) and baselineadjusted performances (right block), estimated through the corresponding linear model of each metric. For baseline-adjusted metrics, only the improvement over the baseline is displayed. The ranking, ranging from 1 (best) to 4 (worst) in each row and block, was based on the expected performance, averaged over folds and indicated in parentheses. The colour scale varies between the block row-wise maximum (red) and 0 (blue). 